\title{
Mercado Laboral, Servicios Intensivos en Conocimiento y Género: Análisis para el Caso Argentino
}

\author{
Lizzie Marcel ${ }^{1}$, Juan Manuel Rodriguez Repeti ${ }^{*}$
}

\begin{abstract}
Resumen: La literatura de género estudia de manera exhaustiva las brechas de género en términos de ingresos, y la desigualdad en las oportunidades de acceso al mercado laboral entre varones y mujeres. Sin embargo, estos estudios se realizan a nivel agregado de la economía, y muy pocas veces, a nivel de industrias. Es por ello, que el estudio de las brechas de género en términos salariales y de acceso laboral desde una perspectiva de sectores productivos es relevante. Más aún, dicho estudio en los sectores de la economía con mayor remuneración. Debido a esto, el presente trabajo pretende enfocarse en el estudio del acceso femenino en el mercado laboral de los Servicios Intensivos en Conocimiento, ya que las tareas que se realizan en estos sectores de la economía son las de remuneraciones más altas. Mediante los datos de la Encuesta Permanente de Hogares de Argentina para el cuarto trimestre de 2020, se encuentra que la probabilidad de la inserción laboral en los Servicios Intensivos en Conocimiento es mayor para los varones que para las mujeres, aunque la participación es heterogénea entre los diferentes sectores.
\end{abstract}

Palabras Clave: Mercado Laboral; Servicios Intensivos en Conocimiento; Género; Brechas de ingreso

\begin{abstract}
The gender literature exhaustively studies gender gaps in terms of income and inequality in the opportunities of access to the labor market between men and women. However, these studies are carried out at the aggregate level of the economy and very rarely at the level of industries. That is why the study of gender gaps in wages and access to employment from a productive sector perspective is relevant. Furthermore, this study in the sectors of the economy with the highest remuneration. Due to this, the present work aims to focus on the study of female access to the labor market of Knowledge-Intensive Services, since the tasks performed in these sectors of the economy are those with the highest remuneration. Using data from the Permanent Household Survey of Argentina for the fourth quarter of 2020, it is found that the probability of labor insertion in Knowledge-Intensive Services is higher for men than for women, although participation is heterogeneous among the different sections.
\end{abstract}

Keywords: Labor Market; Knowledge-Intensive Services; Gender; Income gaps

Submitted: August 23rd $2021 /$ Approved: October $26^{\text {th }}, 2021$

\section{Introducción}

En las últimas décadas se puede observar un cambio a nivel global en la composición de la demanda agregada en las economías, ya que el consumo de servicios pasó a tener una ponderación más relevante que el de bienes. La fluctuación en la demanda se puede atribuir a la aparición de la inversión extranjera directa y a las nuevas cadenas globales de valor, las cuales contribuyeron a cambios en las estructuras productivas, en términos de crecimiento económico y generación de puestos laborales. En función de estas modificaciones se destaca un rasgo sustancial en la dinámica del mercado de empleo, el cual está asociado a la necesidad de una mayor calificación de los trabajadores para desempeñar las diferentes actividades que componen los procesos productivos y, además, permite a los individuos acceder a empleos con mayores retribuciones y beneficios laborales.

Con el desarrollo de la producción de servicios, comenzó a tomar mayor peso un subgrupo de estos en los cuales se requiere un mayor nivel de conocimiento para llevar a cabo las tareas productivas, estos se denominan Servicios Intensivos en Conocimiento (SIC o KBIS por sus siglas en inglés). Este subgrupo abarca una amplia heterogeneidad de servicios y tareas, las cuales van desde la manipulación de software, hasta tareas en el ámbito financiero o de investigación y desarrollo (I+D). Además, dentro de la economía en general y de los servicios en particular, los SIC se destacan por el pago de salarios, en promedio, mayores al resto de las actividades productivas. Por lo tanto, el acceso de los individuos al desarrollo de estas actividades les garantiza un salario medio mayor y, en líneas generales, una mejor calidad de vida.

Las características presentadas por los SIC, motivan el estudio de la composición de su demanda laboral, no solo en relación a la educación formal de los individuos que la integran, sino también en otros aspectos. Por ejemplo, las dimensiones respectivas al género que, si bien existen varios trabajos que analizan estos aspectos de manera en general, el estudio de la participación laboral femenina en los SIC es incipiente, particularmente en Argentina, donde no existen trabajos que realicen un análisis empírico acerca de dicha relación. Por lo tanto, el objetivo de este trabajo es brindar desde la perspectiva de género, un análisis cuantitativo del sector SIC, el acceso, la participación y la calidad de los puestos laborales femeninos.

La presente investigación se estructura de la siguiente forma. En primer lugar, se presenta el marco teórico junto con las hipótesis de

(1) Centro de investigaciones Económicas y Sociales (CIEyS), Universidad Nacional de Mar del Plata, Mar del Plata, Argentina.

(2) Instituto Interdisciplinario de Economía Política de Buenos Aires (IIEP-Baires), Universidad de Buenos Aires, Buenos Aires, Argentina.

*Corresponding author: rodriguezrepeti.jm@economicas.uba.ar 
trabajo. Luego, la metodología en relación a la fuente de datos, variables y técnicas econométricas. Posteriormente se presentan los resultados y su discusión, en último lugar se presentan las conclusiones.

\section{La importancia de los servicios, los SIC y su relación con el género}

A lo largo de la historia, los servicios estuvieron asociados a niveles bajos de calificación técnica con respecto a los esquemas de industrias tradicionales. Estos se caracterizaban por ser actividades de baja productividad, poca innovación y carácter no transable. Con la disminución de los costos de transporte, comunicación y coordinación, que permitieron la desagregación espacial de las etapas productivas (Baldwin, 2006) los servicios tomaron mayor protagonismo en las economías y han cobrado una relevancia creciente a nivel mundial. Según datos de $2018^{1}$, representan el 68\% de la producción, el 59\% del empleo y el $50 \%$ de la inversión mundial, mientras que, en los países en desarrollo, representan el 56\% de la producción y el 54\% del empleo, transformándose en una vía clave para la modernización de estas economías (UNCTAD, 2018), al conformar el principal núcleo de generación de innovación y valor agregado, y concentrar la mayor parte del empleo calificado (López \& Ramos, 2013; Shearmur et al., 2015).

Dentro de los servicios existe un subgrupo, los SIC, que basan su producción y desarrollo en la creación, acumulación y diseminación de conocimiento (Amara et al., 2009; Chichkanov et al., 2021; Miles et al., 1995). La provisión de estos implica el desarrollo y utilización de tecnología de frontera, así como también la implementación de mano de obra altamente calificada, es por ello que los SIC son un factor relevante para la innovación y el desarrollo de las economías (Uriona Maldonado et al., 2013). Por ejemplo, Castelacci (2008) remarca que la cercanía de estos servicios con el paradigma tecnológico basado en las TIC, favorece las oportunidades tecnológicas, así como también, la creación de productos y servicios avanzados relacionados con la Investigación y Desarrollo (I+D). Es por esto que, en los últimos años, los SIC han experimentado una evolución constante tanto en países desarrollados como en vías de desarrollo, explicada en parte por sus características innovadoras y el impulso al crecimiento de otros sectores de la economía (Chichkanov et al., 2021; Miles, 2005; Uriona Maldonado et al., 2013). Según Romero Amado et al. (2018), Argentina cuenta con más cantidad de trabajadores del conocimiento en promedio que el promedio mundial y el de América Latina, dada la importancia de la educación superior en el país, lo cual motiva este trabajo de investigación.

Existe una gran heterogeneidad en las industrias y en la composición laboral en estos servicios (López \& Niembro, (2019) y Romero Amado et al., (2018)). Si bien se demuestra que la remuneración dentro de los SIC es mayor para los varones que para las mujeres, existe escasa evidencia sobre brechas salariales de género en el sector (DueñasFernández et al., 2015). Dueñas-Fernández et al. (2015) encuentran que la inserción en los SIC puede morigerar la brecha salarial pero no reduce completamente la inequidad de género en el mercado laboral español. Una explicación de los autores sugiere que las estructuras sociales y económicas se reflejan también en estos sectores intensivos. Dicho trabajo apunta al estudio de la brecha salarial, lo cual, si bien dista del objetivo de la presente investigación, es un puntapié sobre la cuestión de género en el sector SIC. Por lo tanto, en la literatura se destaca que la mera inserción no reduce las brechas. En términos de resultados, una parte de la diferencia puede explicarse por desigualdad de oportunidades, no obstante, es complejo realizar una distinción entre igualdad de oportunidades y de resultados. Los motivos de las diferencias que distinguen las hipótesis y teorías económicas sobre el tema (Akerlof \& Kranton, 2000; Arrow, 1973; Becker, 1957, 1965; Bertrand, 2010) apuntan tanto a diferencias de habilidades (capital humano) como a imposición de roles, prejuicios y discriminación.

En América Latina, en términos de capital humano, si bien las mujeres están más educadas que los varones, hay diferencias en las habilidades cognitivas y una tendencia en la educación de los varones hacia las ciencias y la informática (Gasparini \& Marchionni, 2015; Marchionni et al., 2019) . Entonces, si bien las mujeres pueden formar parte de sectores que están asociados a nuevas tecnologías, pueden verse discriminadas o segregadas, al cobrar menores salarios o tener un menor acceso a puestos jerárquicos, lo que se conoce como techo de cristal(OECD, 2007).

Dada la creciente participación laboral femenina en Argentina $^{2}$, el sector intensivo en conocimiento presenta potencialidades para las mujeres, ya que, tal como se mencionó, el género femenino muestra ventajas comparativas respecto al masculino en la formación académica (Goldin, 2006), requisito fundamental para el desarrollo de actividades vinculadas a los SIC. Por lo tanto, el cambio en la demanda agregada que deriva en un crecimiento del sector servicios podría incrementar la incorporación de mujeres en estos puestos de trabajo.

Martínez et al. (2020) tomaron en cuenta el sexo de los individuos como control en una regresión logística para analizar si este era determinante en cuanto al acceso a puestos laborales SIC en la Ciudad de Buenos Aires. Si bien encuentran que existen diferencias por género, esta investigación no realiza un estudio exhaustivo de las posibilidades de inclusión femenina en la mano de obra SIC. Vale mencionar que no se encontraron trabajos de investigación que evalúen la relación, siendo la presente investigación un aporte innovador y un posible impulsor para la profundización de futuras investigaciones en este sentido. Para ello se plantean dos hipótesis que guiarán la presente investigación: (i) H1) La participación laboral en los SIC difiere entre mujeres y varones, ceteris paribus; (ii) H2) Existe un diferencial por género entre los sectores, ceteris paribus.

${ }^{1}$ Datos de la Organización Internacional del Trabajo. Ver: https://ilostat.ilo.org/es/topics/women/

${ }^{2}$ Datos de la Organización Internacional del Trabajo. Ver: https://ilostat.ilo.org/es/topics/women/ 


\section{Metodología}

A partir de los datos suministrados por la Encuesta Permanente de Hogares (EPH), realizada por el Instituto Nacional de Estadísticas y Censos (INDEC), para el cuarto trimestre de 2020, se identifican las diferentes actividades según la Clasificación de Actividades Económicas para Encuestas Sociodemográficas (CAES). Además, se utiliza la clasificación de Eurostat (2016) para armar las diferentes categorías
SIC (Tabla 1), permitiendo con esto dividir la muestra de la EPH en actividades productoras de bienes, de servicios intensivos en conocimiento y de otros servicios (e.g. comercio) (NSIC). Realizada esta categorización, se procedió a utilizar métodos estadísticos básicos como tablas de contingencia y pruebas chi-cuadrado, con el fin de realizar un análisis descriptivo de la muestra, en cuanto a composición sectorial, porcentaje de mujeres empleadas, ingresos en cada actividad, entre otros. Tabla 1. Clasificación de Actividades SIC

Tabla 1: Clasificación de Actividades SIC

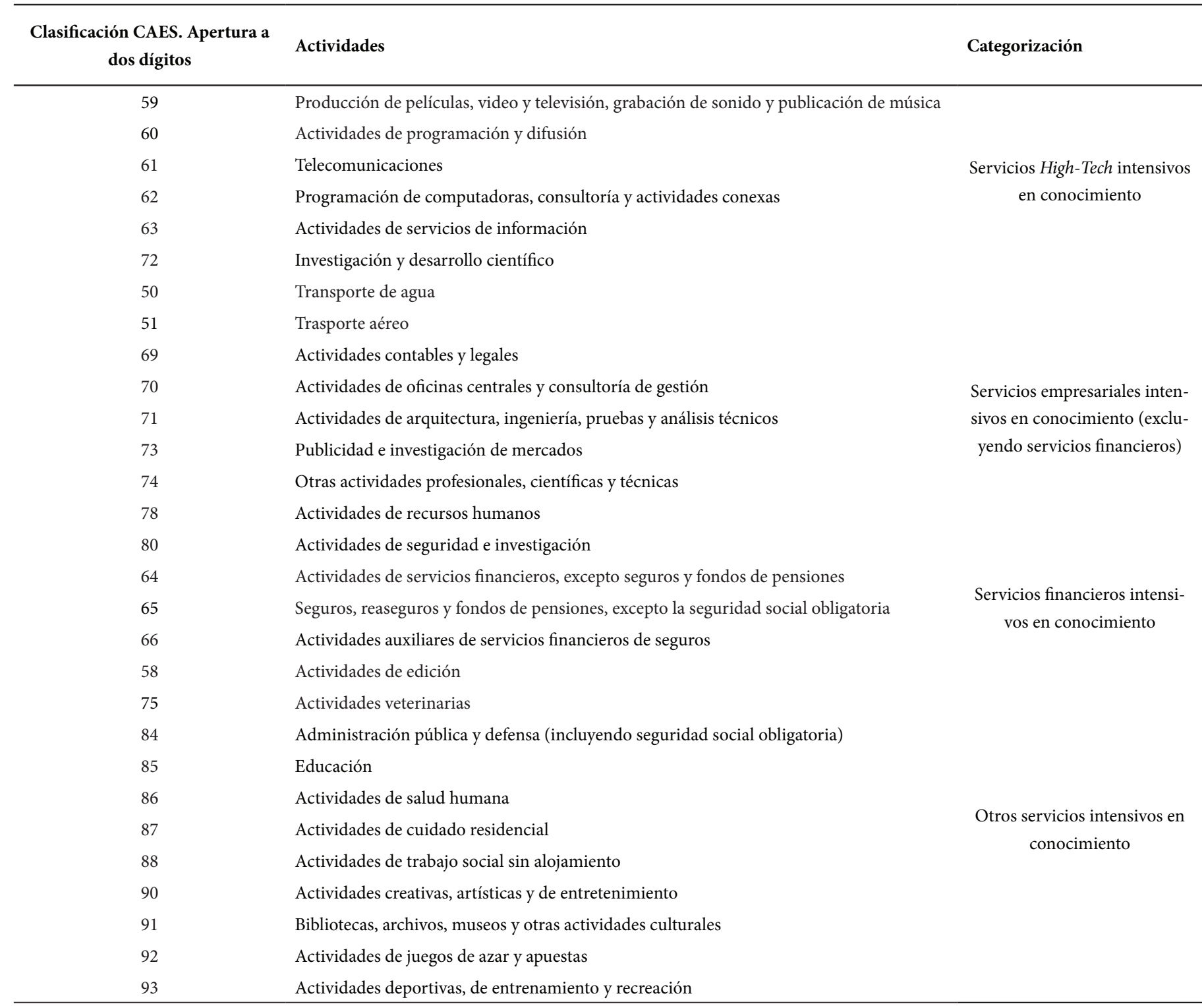

Fuente: Eurostat (2016)

Posteriormente, se opta por modelar un Modelo Lineal Generalizado (MLG) con enlace logit para estimar la probabilidad de pertenencia de las mujeres a un sector particular. Los MLG son modelos lineales para la media transformada de una variable que tiene una distribución de probabilidad en la familia exponencial uniparamétrica (Gill, 2000). Dado que la variable de interés es el sector categorizada como una variable binaria, el MLG con enlace logit se estima por máxima verosimilitud. Los estimadores de máxima verosimilitud (EMV) son consistentes, tienen varianza mínima y se distribuyen normalmente en muestras grandes (Stock et al., 2012; Verbeek, 2004). Los coeficientes estimados, una vez exponenciados, se interpretan como cocientes de chances condicionales en una regresión múltiple. En los modelos 
no lineales, la inferencia estadística debe realizarse mediante pruebas de Wald y Razón de verosimilitud (LR). En cuanto a la capacidad predictiva del modelo, se pueden analizar dos herramientas tales como la tabla de clasificación y la curva ROC. Esta última es un gráfico de la sensibilidad en función de "1-especificidad" para todos los posibles puntos de corte, cuya forma cóncava une las coordenadas $(0,0)$ y $(1,1)$ (Agresti, 2007). Para una especificidad dada, se prefiere una mayor sensibilidad, es decir, cuanto mejor sea el poder predictivo del modelo, más alta será la curva ROC. De esta forma, cuanto mayor es el área debajo de la curva, mejor es la capacidad predictiva del modelo. La curva ROC constituye una herramienta que brinda mayor información que las tablas de clasificación porque resume el poder predictivo del modelo para todos los puntos de corte que puedan ser considerados, por lo tanto, en este trabajo se presenta junto con los modelos el área debajo de la curva como indicador de su capacidad predictiva.

Se formulan tres modelos para analizar la probabilidad de inserción de las mujeres en los SIC intensivos en conocimiento (high-tech, empresariales y financieros), en otros SIC y en el sector de bienes (cada variable dependiente es una binaria que indica si pertenece al sector correspondiente). El efecto de interés está mediado por el parámetro $\beta_{1}$. Se controla por el nivel educativo (variable con distintas categorías por nivel: primario incompleto, completo, secundario incompleto y completo, superior universitario incompleto y completo, y sin instrucción), la nacionalidad (variable binaria que determina si es argentinx o inmigrante) y la region.

logit $(\mu)=\beta_{0}+\beta_{1}$ género $_{i}+\beta_{2}$ nivel $_{\text {educativo }_{i}}+\beta_{3}$ nacionalidad $_{i}+\beta_{4}$ region $_{i}$

\section{Resultados}

\section{Análisis descriptivo}

En primer lugar, presentamos la distribución de los sectores, es decir, el porcentaje de individuos que están empleados en cada sector (Gráfico 1). Un 80\% de los individuos está empleado en el sector de servicios no intensivos en conocimiento. Un $12 \%$ pertenece a otros servicios intensivos en conocimientos, tales como administración pública, educación, actividades de salud humana, de cuidado residencial, artísticas, creativas, entre otras. Mientras que un $5 \%$ se encuentra en la industria manufacturera, sólo un $1 \%$ corresponde a servicios high- tech, empresariales y financieros intensivos en conocimiento.

Gráfico 1: Individuos empleados por sectores

$=$ NSIC

- Servicios high-tech intensivos en conocimiento

- Servicios empresariales intensivos en conocimiento

= Servicios financieros intensivos en conocimiento

- Otros servicios intensivos en conocimiento

$=$ Bienes

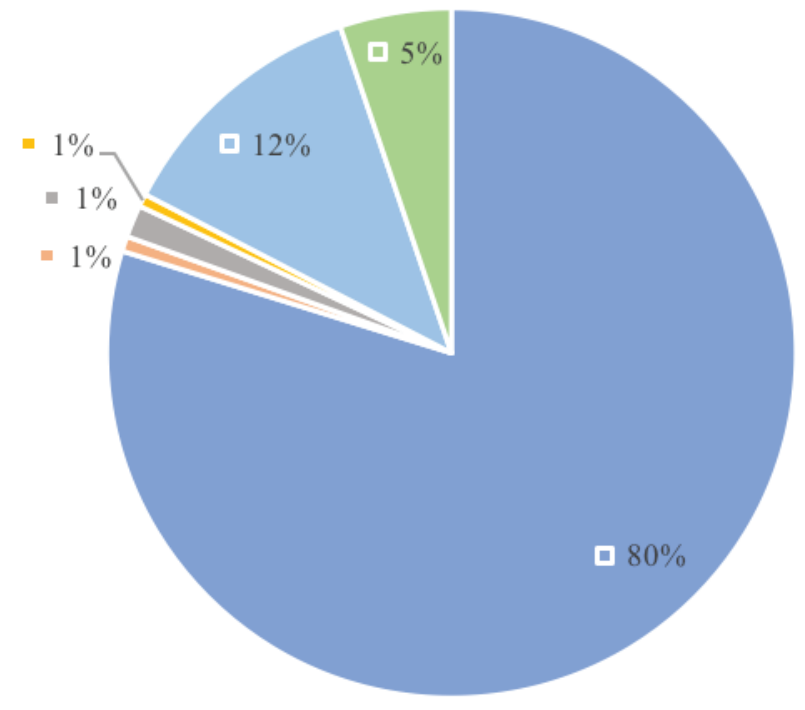

Fuente: Elaboración propia.

En la muestra, un 51\% son mujeres y un $49 \%$ varones $^{3}$. La tasa de actividad difiere entre varones y mujeres, siendo más alta para los varones (68,4\% y $47,6 \%$, respectivamente), mientras que la tasa de empleo es de $61,4 \%$ y $41,9 \%$, respectivamente (Shokida et al., 2021).
Con respecto a la educación, hay proporcionalmente más mujeres que tienen estudios superiores universitarios incompletos y completos (Tabla 2). Mientras que hay proporcionalmente más varones que tienen estudios primarios incompletos y secundarios incompletos y

\footnotetext{
${ }^{3}$ Se entiende que el género discrepa del sexo, ya que el género es como una persona percibe su sexualidad y el sexo es binario. El estudio realizado se enfoca en el sexo y no en el género ya que no se releva este dato en la encuesta.
} 
completos. Así, las mujeres se educan en mayor proporción que los varones, lo cual se entiende como una ventaja relativa que tienen las mujeres en estos términos. Esperaríamos que el acceso de ellas a los sectores intensivos en conocimiento sea mayor. Sin embargo, dado que las mujeres están más expuestas a la desocupación y subocupación horaria (INDEC, 2021) esto podría explicar, en parte, una menor participación en estos sectores intensivos. En el periodo considerado, la tasa de desocupación es de $11,9 \%$ para las mujeres y $10,2 \%$ para los varones, mientras que la tasa de subocupación es de 17,8\% y 13\%, respectivamente (Shokida et al., 2021). Por otra parte, la propia educación podría dar cuenta de una menor inserción en el mercado de trabajo, dado el tiempo que demandan los estudios superiores.

Tabla2: Porcentaje de mujeres y varones por nivel educativo. Total de Aglomerados Urbanos

\begin{tabular}{lcc}
\hline Nivel educativo & Mujer & Varón \\
\hline Primario incompleto & $49,6 \%$ & $50,4 \%^{*}$ \\
Primario completo & $52,6 \%$ & $47,4 \%$ \\
Secundario incompleto & $47,4 \%$ & $52,6 \%^{*}$ \\
Secundario completo & $51,2 \%$ & $48,8 \%{ }^{*}$ \\
Superior universitario incompleto & $55,9 \%^{*}$ & $44,1 \%$ \\
Superior universitario completo & $61,9 \%^{*}$ & $38,1 \%$ \\
Sin instrucción & $49,2 \%$ & $50,8 \%^{*}$ \\
\hline
\end{tabular}

Fuente: Elaboración Propia en base a datos de la EPH.

${ }^{*}$ Residuos ajustados estandarizados $>2$ en v.a. Prueba chi-cuadrado.

En la Tabla 3 presentamos la proporción de mujeres y varones por rama de actividad. Hay proporcionalmente más varones en bienes, high tech, financieros y empresariales, mientras que hay proporcionalmente más mujeres que se encuentran en servicios que no son intensivos en conocimiento y en otros servicios intensivos en conocimiento. Esto tiene concordancia con las actividades que se agrupan dentro de este último subsector, tales como la enseñanza, actividades veterinarias, de salud humana, cuidado residencial, trabajo social, creativas, artísticas y de entretenimiento, entre otros, ámbitos en los que la tasa de feminización es más alta (Shokida et al., 2021).
Tabla 3: Porcentaje de mujeres y varones por rama de actividad. Total de Aglomerados Urbanos

\begin{tabular}{lll}
\hline Rama de actividad & Mujer & Varón \\
\hline Bienes & $29,8 \%$ & $70,2 \%$ \\
Servicios high-tech intensivos en conocimiento & $28,2 \%$ & $71,8 \%$ \\
Servicios financieros intensivos en conocimiento & $43,8 \%$ & $56,2 \%$ \\
Servicios empresariales intensivos en conocimiento & $39,4 \%$ & $60,6 \%$ \\
NSIC & $53,1 \%$ & $46,9 \%$ \\
Otros servicios intensivos en conocimiento & $58,8 \%$ & $41,2 \%$ \\
\hline
\end{tabular}

Fuente: Elaboración Propia en base a datos de la EPH.

${ }^{*}$ Residuos ajustados estandarizados $>2$ en v.a. Prueba chi-cuadrado.

En la Tabla 4 presentamos el ingreso medio para los individuos ocupados por rama de actividad. En primer lugar, podemos observar que el ingreso es sensiblemente más alto en los SIC que en bienes o servicios no intensivos en conocimiento, en particular, dentro del mismo subsector difieren. Así, los servicios intensivos financieros son los de mayor peso relativo en términos de ingresos y le siguen los high-tech. Por otro lado, podemos analizar que el ingreso medio de los varones es mayor en todos los sectores en comparación con las mujeres. Si nos detenemos en las mujeres ocupadas, aquellas que se encuentran en el SIC financiero son las que tienen un ingreso promedio más alto y, por lo tanto, la brecha de ingresos entre mujeres y varones es la más baja $(3,12 \%)^{4}$. El segundo subsector de mayores ingresos para ellas es el de otros SIC, lo cual podría explicarse por la mayor proporción de mujeres en dicho sector, tal como presentamos en la Tabla 3. Sin embargo, en este subsector las mujeres ganan, en promedio, un 11,65\% menos que los varones. Dentro de los SIC, la brecha más grande la encontramos para los high-tech, en el cual, las mujeres ganan $27,76 \%$ menos, en promedio, que los varones. El menor ingreso medio lo encontramos en otros servicios no intensivos en conocimiento, tanto para mujeres como para varones. Adicionalmente, realizamos una prueba estadística para comparar el ingreso por género y concluimos que el ingreso medio difiere entre mujeres y varones ${ }^{5}$.

Tabla 4: Ingreso medio de ocupados y ocupadas por rama de actividad. Total de Aglomerados Urbanos

\begin{tabular}{|c|c|c|c|c|}
\hline \multirow{2}{*}{ Rama de actividad } & \multicolumn{4}{|c|}{ Ingreso medio } \\
\hline & Solo Mujeres & Solo varones & Total & Brecha \\
\hline Bienes & 18624,02 & 31846,11 & 27758,21 & $41,52 \%$ \\
\hline Servicios financieros intensivos en conocimiento & 44329,26 & 45754,61 & 45069,69 & $3,12 \%$ \\
\hline Servicios empresariales intensivos en conocimiento & 29937,37 & 34356,81 & 32403,04 & $12,86 \%$ \\
\hline NSIC & 14256,58 & 21003,58 & 18375,71 & $32,12 \%$ \\
\hline Observaciones & 7612 & 10017 & 17629 & \\
\hline
\end{tabular}

Fuente: Elaboración Propia en base a datos de la EPH.

${ }^{4} \mathrm{La}$ brecha se calcula en términos del ingreso más alto.

${ }^{5}$ Con un estadístico F igual a 1,8919 se rechaza la hipótesis nula de igualdad de medias al nivel de significatividad dado, en favor de la hipótesis alternativa. 
Si en lugar de analizar el ingreso promedio de bolsillo, comparamos el ingreso horario, es decir, el ingreso medio por hora por sector entre mujeres y varones, observamos que las brechas no se modifican sensiblemente (Tabla 5). En el caso de todos los sectores analizados, las mujeres ganan por hora menos que los varones en promedio, no obstante, las brechas de los sectores que demandan mayor conocimiento son mayores en términos porcentuales dado que las mujeres trabajan menos horas que los varones porque dedican gran parte del tiempo al trabajo doméstico y de cuidados no remunerado ${ }^{6}$ (Shokida et al.,
2021) e incluso se puede argüir que este resultado se relaciona con la participación de las mujeres en dichos sectores. Con respecto a otros sectores, las brechas se reducen por el mayor porcentaje de mujeres en actividades como otros SIC y servicios que no son intensivos en conocimiento. En síntesis, si bien la participación de las mujeres en algunos subsectores es alta, las brechas de ingresos persisten y reflejan las diferencias de género en el mercado trabajo. Tabla 5. Ingreso horario medio de ocupados y ocupadas por rama de actividad. Total de Aglomerados Urbanos.

Tabla 5: Ingreso horario medio de ocupados y ocupadas por rama de actividad. Total de Aglomerados Urbanos

\begin{tabular}{|c|c|c|c|c|}
\hline \multirow{2}{*}{ Rama de actividad } & \multicolumn{4}{|c|}{ Ingreso horario medio } \\
\hline & Solo Mujeres & Solo varones & Total & Brecha \\
\hline Bienes & 132,94 & 185,06 & 169,00 & $28,16 \%$ \\
\hline Servicios high-tech intensivos en conocimiento & 191,13 & 299,02 & 271,68 & $36,08 \%$ \\
\hline Servicios financieros intensivos en conocimiento & 250,32 & 274,91 & 263,69 & $8,94 \%$ \\
\hline Servicios empresariales intensivos en conocimiento & 208,69 & 230,81 & 220,94 & $9,58 \%$ \\
\hline NSIC & 129,07 & 133,10 & 131,56 & $3,03 \%$ \\
\hline Otros servicios intensivos en conocimiento & 303,16 & 320,34 & 309,75 & $5,36 \%$ \\
\hline
\end{tabular}

Fuente: Elaboración Propia en base a datos de la EPH.

\section{Resultados de la Estimación}

En la Tabla 6 se presentan los resultados de los modelos logit por sector agrupado. Estos modelos apuntan a estimar la probabilidad de que un individuo se encuentre en un sector en particular. El interés radica en el parámetro asociado a la variable género que indicaría diferencias en la probabilidad de acceso entre mujeres y varones. En la Tabla se pueden observar los valores de los coeficientes junto con los errores estándar y el nivel de significatividad ${ }^{7}$.

El valor de probabilidad de las pruebas LR menor al $1 \%$ indica que en los modelos existe un cambio significativo en la función de log verosimilitud cuando pasamos de un modelo nulo al modelo corriente. Por lo tanto, los modelos son globalmente significativos.

Tabla 6: Resultados de la estimación.

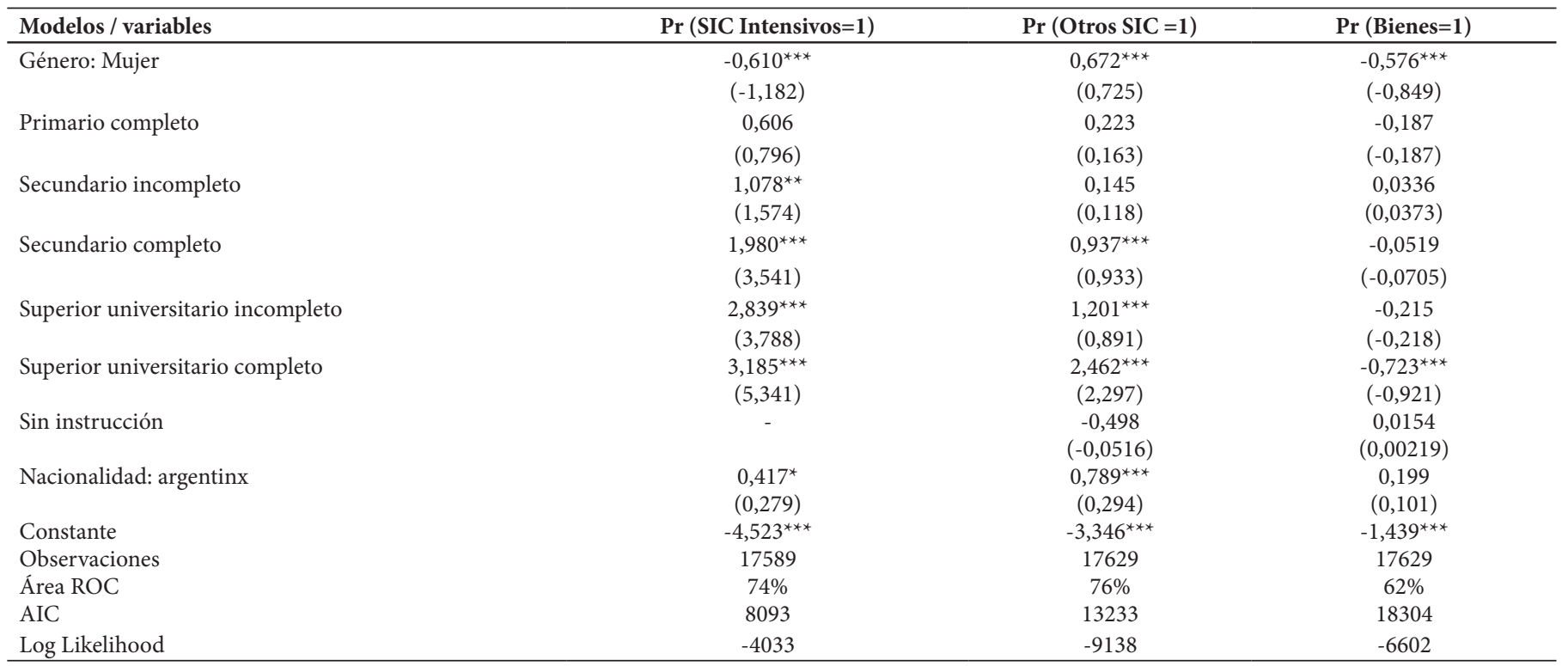

Fuente: Elaboración propia en base a datos de la EPH.

Normalized beta coefficients in parentheses

${ }^{* * *} \mathrm{p}<0.01,{ }^{* *} \mathrm{p}<0.05,{ }^{*} \mathrm{p}<0.1$

Notas: (i) Se estima para individuos ocupados; (ii) Se controla por región.

${ }^{6}$ Se estimaron los mismos modelos con datos de la EPH del último trimestre de 2019 y no se encuentran diferencias con los resultados obtenidos.

${ }^{7}$ Se estimaron los mismos modelos con datos de la EPH del último trimestre de 2019 y no se encuentran diferencias con los resultados obtenidos. 
Tabla 7: Cocientes de chances condicionales

\begin{tabular}{lccc}
\hline Modelos $/$ variables & Pr (SIC Intensivos=1) & $\operatorname{Pr}($ Otros SIC=1) & Pr(Bienes=1) \\
Género: Mujer & 0,54 & 1,96 & 0,56 \\
Primario completo & 1,83 & 1,25 & 0,83 \\
Secundario incompleto & 2,94 & 2,55 & 0,95 \\
Secundario completo & 7,24 & 3,32 & 0,81 \\
Superior universitario incompleto & 17,10 & 11,73 & 0,49 \\
Superior universitario completo & 24,17 & 0,61 & 1,02 \\
Sin instrucción & - & 2,20 & 1,22 \\
Nacionalidad: argentinx & 1,52 & & \\
\hline
\end{tabular}

Fuente: Elaboración propia en base a datos de la EPH.

Con respecto al primer modelo, la variable de género es estadísticamente significativa y presenta el signo esperado, por lo tanto, permite validar la H1). En los SIC intensivos en conocimiento, dentro del cual se encuentran high-tech, empresariales y financieros, la probabilidad de inserción de las mujeres es de un 46\% menos en comparación con los varones (ver Tabla 7). Asimismo, se destacan los niveles de educación que aumentan la probabilidad de pertenecer a estos servicios intensivos, donde, si bien el coeficiente de secundario incompleto es estadísticamente significativo, el efecto marginal más alto se encuentra en el estudio superior universitario completo, para el cual, los individuos que tienen este nivel educativo tienen 23 veces más de chances de pertenecer a este subsector que individuos con primario incompleto ${ }^{8}$. En este modelo se controla por nacionalidad y por región.

En el segundo modelo, se puede observar que la variable de género es estadísticamente significativa y presenta el signo esperado de acuerdo a lo comentado en el análisis descriptivo. La probabilidad de inserción de las mujeres en otros SIC es de un 95\% más que los varones, esto se puede explicar por la alta participación de las mujeres en estos servicios en particular que engloban actividades como la educación, el cuidado residencial, la salud humana y el trabajo social, entre otros. Nuevamente, los niveles educativos más altos son relevantes para explicar la probabilidad de emplearse en el sector. En este modelo se controla por nacionalidad y por región.

Por último, el tercer modelo también presenta resultados interesantes en concordancia con el resultado descriptivo. En el sector de bienes, la probabilidad de inserción de las mujeres es significativamente menor, comparada con los varones. Así, las mujeres tienen un $44 \%$ menos de probabilidad de estar empleadas en el sector en relación a los varones. La industria manufacturera es uno de los sectores con la tasa más baja de feminización de la economía, junto con el transporte y la construcción (Shokida et al., 2021), por lo tanto, esto puede explicar estas diferencias. Asimismo, la industria es un sector con calificación más baja en relación a los servicios y con técnicas más operativas y tareas manuales, lo cual, se entiende como actividades tradicionalmente asociadas al género masculino (Goldin, 2006). Este resultado representa esos estereotipos de género en cuanto a las tareas que desarrollan mujeres y varones.
En este modelo se controla por nacionalidad y por región.

Los resultados permiten validar las dos hipótesis de investigación, por un lado, la probabilidad de inserción en los SIC difiere entre varones y mujeres, y la participación de las mujeres es diferente entre sectores. Esta distinción marcada en el acceso a distintos sectores de la economía refuerza los estereotipos de género. Por lo tanto, si bien lo que representan los modelos es una situación en un momento particular, en términos de habilidades y conocimientos, hay un campo de acción para las mujeres en los servicios intensivos. Sin embargo, detrás de esta inserción pueden operar diversas cuestiones tales como la discriminación y la segregación hacia las mujeres en los sectores que mejor pagan, incluso antes de ingresar.

\section{Conclusiones}

Este trabajo realiza un aporte preliminar en términos de la inserción de las mujeres en los sectores intensivos en conocimiento, que presentan una potencialidad creciente en la economía. Apuntalar y disipar la segregación en el mercado laboral son puntos fundamentales para las políticas públicas en materia de igualdad de género, en vías de lograr uno de los objetivos en la agenda de desarrollo sostenible (PNUD, 2017). Según los datos presentados se puede concluir que para el caso argentino, si bien las mujeres poseen más educación formal que los varones, esto no garantiza su inserción en los sectores que requieren mayor conocimiento. Lo que fundamenta una disparidad o diferencia de oportunidades para adquirir mejores empleos según el sexo del individuo que busque trabajo. Debido a esto, se entiende que el estudio de las oportunidades de acceder al mercado laboral SIC por parte de las mujeres y las desigualdades que se generan en esto debe ser estudiado con mayor profundidad con el fin de dar luz a esta situación, y en última instancia que se puedan realizar recomendaciones para equiparar oportunidades entre varones y mujeres. Expuesto esto, las dos hipótesis planteadas en este trabajo se verifican, ya que queda demostrado que las mujeres poseen menor posibilidad de emplearse en el sector SIC. Y, si lo hacen, se emplean dentro de los servicios que presentan menor remuneración (otros SIC), en donde predominan actividades como la educación y salud.

\footnotetext{
${ }^{8}$ El efecto es alto por la categoría base empleada.
} 


\section{Referencias}

Agresti, A. (2007). An introduction to categorical data analysis (2nd ed). Wiley-Interscience.

Akerlof, G. A., \& Kranton, R. E. (2000). Economics and identity. The quarterly journal of economics, 115(3), 715-753.

Amara, N., Landry, R., \& Doloreux, D. (2009). Patterns of innovation in knowledge-intensive business services. The Service Industries Journal, 29(4), 407-430. https://doi.org/10.1080/02642060802307847

Arrow, K. (1973). The Theory of Discrimination. Princeton University, Department of Economics, Industrial Relations Section. https:// econpapers.repec.org/paper/priindrel/30a.htm

Baldwin, R. E. (2006). Globalisation: The great unbundling (s). Economic council of Finland.

Becker, G. S. (1957). The economics of discrimination: An economic view of racial discrimination. University of Chicago.

Becker, G. S. (1965). A Theory of the Allocation of Time. The economic journal, 75(299), 493-517.

Bertrand, M. (2010). New Perspectives on Gender. En Handbook of Labor Economics (Vol. 4B, pp. 1543-1590). Elsevier. https://ideas.repec.org/h/eee/labchp/5-17.html

Castellacci, F. (2008). Technological paradigms, regimes and trajectories: Manufacturing and service industries in a new taxonomy of sectoral patterns of innovation. Research Policy, 37(6-7), 978-994. https://doi.org/10.1016/j.respol.2008.03.011

Chichkanov, N., Miles, I., \& Belousova, V. (2021). Drivers for innovation in KIBS: Evidence from Russia. The Service Industries Journal, 41(7-8), 489-511. https://doi.org/10.1080/02642069.2019.1570151

Dueñas-Fernández, D., Iglesias-Fernández, C., \& Llorente-Heras, R. (2015). Is there less gender inequality in the service sector? The gender wage-gap in knowledge-intensive services. Social Science Information, 54(3), 369-393. https://doi.org/10.1177/0539018415586216

Eurostat. (2016). Glossary:Knowledge-intensive services (KIS). https://ec.europa.eu/eurostat/statistics-explained/index. php?title=Glossary:Knowledge-intensive_services_(KIS)

Gasparini, L., \& Marchionni, M. (2015). ¿ Brechas que se cierran. Aumento y desaceleración de la participación laboral femenina en América Latina. Centro de Estudios Distributivos, Laborales y Sociales de la Universidad Nacional de la Plata. Buenos Aires: UNPL.

Gill, J. (2000). Generalized linear models: A unified approach. Sage Publications, Inc.

Goldin, C. (2006). The quiet revolution that transformed women's employment, education, and family. American economic review, 96(2), 1-21.
INDEC. (2021). Dosier estadístico en conmemoración del $110^{\circ}$ Día Internacional de la Mujer (p. Dosier estadístico; pp. 1-32). https://www.indec. gob.ar/ftp/cuadros/publicaciones/dosier_estadistico_8M_2021.pdf

López, A., \& Niembro, A. (2019). La Heterogeneidad de los Servicios Intensivos en Conocimiento: El Caso de Argentina. Journal of technology management \& innovation, 14(4), 85-99.

López, A., \& Ramos, D. (2013). Los servicios basados en el conocimiento: Una nueva oportunidad exportadora para la Argentina'. Documento de trabajo, 53.

Marchionni, M., Gasparini, L., \& Edo, M. (2019). Brechas de género en América Latina. Un estado de situación. CAF.

Martínez, R. G., Leone, J. G., \& Rodriguez-Repeti, J. M. (2020). Morfología del empleo en las industrias de servicios intensivos en conocimiento. El caso de la Ciudad Autónoma de Buenos Aires. Revista Escuela de Administración de Negocios.

Miles, I. (2005). Knowledge intensive business services: Prospects and policies. Foresight, 7(6), 39-63. https://doi.org/10.1108/14636680510630939

Miles, I., Kastrinos, N., Flanagan, K., Bilderbeek, R., Den Hertog, P., Huntink, W., \& Bouman, M. (1995). Knowledge-Intensive Business Services: Users. Carriers and Sources of Innovation, EIMS publication, 15.

OECD. (2007). ICTs and Gender (OECD Digital Economy Papers N. 129 ; OECD Digital Economy Papers, Vol. 129). https://doi. org/10.1787/231011217663

PNUD. (2017). Informe Nacional sobre Desarrollo Humano 2017. Información para el desarrollo sostenible: Argentina y la Agenda 2030.

Romero Amado, J., López Toache, V., \& Sánchez Daza, G. (2018). Las condiciones laborales en la industria de los servicios empresariales intensivos en conocimiento (SEIC) en México en el nuevo siglo. Análisis económico, 33(83), 145-175.

Shearmur, R., Doloreux, D., \& Laperrière, A. (2015). Is the degree of internationalization associated with the use of knowledge intensive services or with innovation? International Business Review, 24(3), 457-465. https://doi.org/10.1016/j.ibusrev.2014.10.004

Shokida, N., Serpa, D., Domenech, L., Moure, J., Fernández, E. M., Espiñeira, L., \& Santellán, C. (2021). Ecofeminita/EcoFemiData: Informes ecofemidata (v1.0). Zenodo. https://doi.org/10.5281/ZENODO.4540185

Stock, J. H., Watson, M. W., \& Arrazola Vacas, M. (2012). Introducción a la econometría (3a. Ed.). Pearson Educación.

UNCTAD (Ed.). (2018). Investment and new industrial policies. United Nations.

Uriona Maldonado, M., Morero, H., \& Borrastero, C. (2013). Catching up en Servicios Intensivos en conocimiento: El caso de la producción de software y servicios informáticos de Argentina y Brasil. https:// ri.conicet.gov.ar/handle/11336/8324

Verbeek, M. (2004). A guide to modern econometrics. John Wiley \& Sons. 
